\title{
Revised equipartition \& minimum energy formula for magnetic field strength estimates from radio synchrotron observations
}

\author{
RAINER BECK and MARITA KRAUSE \\ Max-Planck-Institut für Radioastronomie, Auf dem Hügel 69, D-53121 Bonn, Germany
}

Received 19 April 2005; accepted 25 May 2005; published online 1 July 2005

\begin{abstract}
The commonly used classical equipartition or minimum-energy estimate of total magnetic fields strengths from radio synchrotron intensities is of limited practical use because it is based on the hardly known ratio $\mathcal{K}$ of the total energies of cosmic ray protons and electrons and also has inherent problems. We present a revised formula, using the number density ratio $\mathbf{K}$ for which we give estimates. For particle acceleration in strong shocks $\mathbf{K}$ is about 40 and increases with decreasing shock strength. Our revised estimate for the field strength gives larger values than the classical estimate for flat radio spectra with spectral indices of about $0.5-0.6$, but smaller values for steep spectra and total fields stronger than about $10 \mu \mathrm{G}$. In very young supernova remnants, for example, the classical estimate may be too large by up to $10 \times$. On the other hand, if energy losses of cosmic ray electrons are important, $\mathbf{K}$ increases with particle energy and the equipartition field may be underestimated significantly. Our revised larger equipartition estimates in galaxy clusters and radio lobes are consistent with independent estimates from Faraday rotation measures, while estimates from the ratio between radio synchrotron and X-ray inverse Compton intensities generally give much weaker fields. This may be explained e.g. by a concentration of the field in filaments. Our revised field strengths may also lead to major revisions of electron lifetimes in jets and radio lobes estimated from the synchrotron break frequency in the radio spectrum.
\end{abstract}

Key words: ISM: magnetic fields - ISM: supernova remnants - galaxies: active - galaxies: clusters: general - galaxies: magnetic fields - radio continuum: general

(c)0000 WILEY-VCH Verlag GmbH \& Co. KGaA, Weinheim

\section{Introduction}

Magnetic fields play an important role in supernova remnants (Fürst \& Reich 2004), in the interstellar medium of galaxies (Beck et al. 1996, Beck 2004), in the intergalactic medium of clusters (Carilli \& Taylor 2002), and in the jets and lobes of active galaxies (Rees 1985, Blandford 2001). The relative importance among the other competing forces can be estimated by comparing the corresponding energy densities or pressures. For most extragalactic objects measurements of the magnetic field strength are based on radio synchrotron emission and Faraday rotation measures (RM) of the polarized radio emission (see Heiles 1976 and Verschuur 1979 for reviews of the observational methods).

Total synchrotron emission traces the total field in the sky plane, polarized synchrotron emission the regular field component. The regular field can be coherent (i.e. preserving its direction within the region of observation) or incoherent (i.e. frequently reversing its direction).

Correspondence to: rbeck@mpifr-bonn.mpg.de
Determination of the field strength from the synchrotron intensity needs information about the number density of the cosmic ray electrons, e.g. via their $\gamma$-ray bremsstrahlung emission or X-ray emission by inverse Compton scattering. If such data are lacking, an assumption about the relation between cosmic ray electrons and magnetic fields has to be made. The most commonly used approaches are:

- Minimum total energy density $\left(\epsilon_{\mathrm{tot}}=\epsilon_{\mathrm{CR}}+\epsilon_{\mathrm{B}}=\min \right)$

- Equipartition between the total energy densities of cosmic rays and that of the magnetic field $\left(\epsilon_{\mathrm{B}}=\epsilon_{\mathrm{CR}}\right)$

- Pressure equilibrium $\left(\epsilon_{\mathrm{B}}=\frac{1}{3} \epsilon_{\mathrm{CR}}\right)$

The minimum-energy and equipartition estimates give very similar results and are often used synonymously. The idea is that cosmic ray particles and magnetic fields are strongly coupled and exchange energy until equilibrium is reached. Deviations from equilibrium occur if, for example, the injection of particles or the generation of magnetic fields is restricted to a few sources or to a limited period. The mean distance between sources and their mean lifetime define the smallest scales in space and time over which equipartition can be assumed to hold. 
The minimum-energy assumption was first proposed by Burbidge (1956) and applied to the optical synchrotron emission of the jet in M87. Since then the validity of this method has been discussed in the literature. Duric (1990) argued that any major deviation from equipartition would be in conflict with radio observations of spiral galaxies.

The azimuthally averaged equipartition strength of the field in the Milky Way and its radial variation (Berkhuijsen, in Beck 2001) agree well with the model of Strong et al. (2000) their Fig. 6) based on radio continuum and $\gamma$ ray surveys and cosmic ray data near the sun. On the other hand, Chi \& Wolfendale (1993) claimed significant deviations from equipartition conditions in the Magellanic Clouds. Pohl (1993b) replied that the standard proton-to-electron ratio used in the equipartition formula may be smaller in the Magellanic Clouds compared to the Milky Way.

Equipartition estimates of field strengths were determined in many spiral galaxies (Beck 2000). The mean strength of the total field ranges from a few $\mu \mathrm{G}$ in galaxies with a low star-formation rate to $\simeq 30 \mu \mathrm{G}$ in grand-design galaxies like M 51. The mean total field strength of a sample of ShapleyAmes galaxies is $9 \mu \mathrm{G}$ (Niklas 1997 see Sect. 4.1). The relation between the total field strength and the star formation rate is deduced from the radio-infrared correlation (Helou \& Bicay 1993, Niklas \& Beck 1997). The total field is strongest in the spiral arms, but the strength of the large-scale regular field in most galaxies is highest in interarm regions (Beck 2000, 2001).

In the radio lobes of strong FRII radio galaxies the equipartition estimates of the field strength are 20-100 $\mu \mathrm{G}$, in hot spots $100-600 \mu \mathrm{G}$, and $0.5-1 \mu \mathrm{G}$ in the intracluster medium of galaxy clusters and in relics (see Sect. 4.3). In these estimates relativistic protons were assumed to be absent ("absolute minimum energy") or contribute similarly to the total energy density as the electrons. The ratio of radio synchrotron to inverse Compton X-ray intensities can be used as another estimate of the field strength (e.g. Carilli \& Taylor 2002). In most radio lobes the two estimates are similar, but there are also significant deviations where "out of equipartition" conditions have been claimed (see Sect. 4.4).

Faraday rotation measures RM are sensitive to the coherent regular field component along the line of sight and to the density of thermal electrons which can be derived from thermal emission or pulsar dispersion measures DM. The ratio $R M / D M$ is a widely used measure of coherent field strengths in the Milky Way. The derived values are lower than the equipartition estimates from polarized intensity (Beck et al. 2003). In galaxy clusters, observations of fluctuations in RM are used to estimate total field strengths. In the Coma cluster such data indicate fields one order of magnitude stronger than the equipartition value (Giovannini et al. 1993, Feretti et al. 1995, Fusco-Femiano et al. 1999, Govoni \& Feretti 2004). Strong total fields have also been claimed from observations of rotation measures in many other clusters (Carilli \& Taylor 2002).

All methods to estimate field strengths are subject to bias. Firstly, a positive correlation between field strength and cosmic ray electron density on small scales, which are unre- solved by the telescope beam or occur along the line of sight, leads to an overestimate of the equipartition strengths of the total and regular field components (Beck et al. 2003). Furthermore, synchrotron intensity is biased towards regions of strong fields, so that the equipartition estimates are higher than the average field strength (Sect. 4.4). Field strengths based on Faraday rotation measures may also be overestimated if small-scale fluctuations in magnetic field and in thermal gas density are positively correlated. Finally, Newman et al. (2002) and Murgia et al. (2004) pointed out that field estimates based on Faraday rotation measures are likely to be too high by a factor of a few if there is a spectrum of turbulence scales.

In this paper we show that there are inherent problems with the classical equipartition / minimum-energy formula, especially when computing its parameters from observable quantities. We present a revised formula which is based on integration of the cosmic ray energy spectrum rather than the radio frequency spectrum and discuss the limitations of its application.

\section{The classical minimum-energy formula}

The classical textbook minimum-energy formula is based on a value for the total cosmic ray energy density which is determined by integrating the radio spectrum from $\nu_{\min }$ to $\nu_{\max }$, usually from $10 \mathrm{MHz}$ to $10 \mathrm{GHz}$, which is the typical range accessible to observations. Particles and magnetic fields are assumed to fill the source homogeneously, and the field is assumed to be completely tangled (no preferred direction). Then the field minimum-energy strength $B_{\min }$ is quoted as (e.g. Pacholcyk 1970, Longair 1994 p. 292; Rohlfs \& Wilson (1996):

$B_{\text {class }}=\left(6 \pi G(\mathcal{K}+1) L_{\nu} / V\right)^{2 / 7}$

where $G$ is a product of several functions varying with $\nu_{\min }$, $\nu_{\max }$ and synchrotron spectral index $\alpha$ (see Eq. A20 for details). $\mathcal{K}$ is the ratio of the total energy of cosmic ray nuclei to that of the synchrotron emitting electrons + positrons. $L_{\nu}$ is the synchrotron luminosity at frequency $\nu, V$ is the source's volume, and $L_{\nu} / V$ is the synchrotron emissivity.

The resulting magnetic energy density $\epsilon_{\mathrm{B}}$ is $3 / 4$ of the cosmic ray energy density $\epsilon_{\mathrm{CR}}$, so that $B_{\text {class }}$ is $8 \%$ smaller than the equipartition field strength $B_{\text {eq,class: }}$ :

$B_{\text {class }} / B_{\text {eq,class }}=(3 / 4)^{2 / 7}$

The problems with the classical formula are the following:

(1) The radio emissivity $L_{\nu} / V$ is the average over the source's volume. Since radio intensity, in the case of equipartition between magnetic fields and cosmic rays, varies with about the fourth power of the total field strength, it emerges mainly from the regions with the highest field strengths, so that $B_{\min }$ is larger than the volume-averaged field strength if the local field strength $B$ is inhomogeneous within the source. This problem can be reduced by replacing $L_{\nu} / V$ by $I_{\nu} / l$, 
where $I_{\nu}$ is the local synchrotron intensity (surface brightness) and $l$ is the pathlength through the emitting medium (see Eq. A20).

(2) A fixed integration interval $\nu_{\min }$ to $\nu_{\max }$ is used in Eq. (1). The critical frequency of synchrotron emission of an electron with energy $E$ in a magnetic field of strength $B$ with a component $B_{\perp}$ perpendicular to the velocity vector is (Lang 1999. p. 29):

$\nu_{\text {crit }}=c_{1} E^{2} B_{\perp}=16.08 \mathrm{MHz}(E / \mathrm{GeV})^{2}\left(B_{\perp} / \mu \mathrm{G}\right)$

where $c_{1}=3 e /\left(4 \pi m_{\mathrm{e}}{ }^{3} c^{5}\right) . e$ is the elementary charge, $m_{\mathrm{e}}$ the electron mass, and $c$ the velocity of light.

Note that the synchrotron emission of a single electron is maximum at the frequency $\nu_{\max }=0.29 \nu_{\text {crit }}$ (Longair 1994 p. 246), but for a continuous energy spectrum of electrons the maximum contribution at a given frequency is emitted by electrons which are a factor of almost two lower in energy (Webber et al. 1980). As a result, Eq. (2) can be used for the maximum synchrotron emission from a spectrum of electrons around $E$.

The standard integration interval of $10 \mathrm{MHz}-10 \mathrm{GHz}$ corresponds to an interval $\left[E_{1}-E_{2}\right]$ in the electron energy spectrum of $800 \mathrm{MeV}-25 \mathrm{GeV}$ in a $1 \mu \mathrm{G}$ field, $250 \mathrm{MeV}-$ $8 \mathrm{GeV}$ in a $10 \mu \mathrm{G}$ field, or to $80 \mathrm{MeV}-2.5 \mathrm{GeV}$ in a $100 \mu \mathrm{G}$ field. The integrated cosmic ray energy $\epsilon_{\mathrm{CR}}$ is proportional to $\left[E_{1}^{1-2 \alpha}-E_{2}^{1-2 \alpha}\right]$ where $\alpha$ is the synchrotron spectral index (see Eq. A5), and $E_{1}$ and $E_{2}$ are fixed integration limits. Replacing $E_{1}$ and $E_{2}$ by $\nu_{\min }$ and $\nu_{\max }$ in the classical minimum-energy formula via Eq. (2) introduces an additional term depending on the magnetic field strength. As a consequence, the total energy $\epsilon_{\text {tot }}$ depends on a constant power of $B$ (see Longair 1994 p. 292) and the wrong derivative $d \epsilon_{\text {tot }} / d B$ leads to the constant exponent $2 / 7$ in Eq. (1). The classical minimum-energy formula is formally incorrect.

(3) $\mathcal{K}$ in Eq. (1) is the ratio of the total energy density of cosmic ray nuclei to that of the electrons (+positrons). Knowledge of $\mathcal{K}$ would require measurements of the spectra of the main cosmic ray species over the whole energy range, especially at low particle energies which, for steep spectra, contribute mostly to the total energy. Instead, the total energy of cosmic ray electrons is approximated in the classical formula by integrating the radio spectrum between fixed frequency limits, and the energy of the cosmic ray nuclei is assumed to scale with $\mathcal{K}$.

This classical procedure is subject to major uncertainties. Firstly, the observable radio spectrum only traces the spectrum of cosmic ray electrons over a small energy range (Eq. (2)). Secondly, the ratio $\mathcal{K}$ of total energies may differ from that in the observable energy range. What would be needed in the classical formula is the energy ratio $\mathcal{K}^{\prime}$ in the limited energy range traced by the observed synchrotron emission. In case of energy losses (Sect. 3.4) $\mathcal{K}^{\prime}$ may deviate strongly from $\mathcal{K}$. As the other input numbers of the classical formula are generally known with sufficient accuracy, the uncertainty in $\mathcal{K}$ is the reason why the equipartition / minimum energy field strengths are regarded as crude estimates and the field estimates are often given with a scaling of $(\mathcal{K}+1)^{2 / 7}$.
We propose to use, instead of the energy ratio $\mathcal{K}$, the $r a$ tio of number densities $\mathbf{K}$ of cosmic ray protons and electrons per particle energy interval within the energy range traced by the observed synchrotron emission. Measurements of the local Galactic cosmic rays near the sun (see Appendix) yield $\mathbf{K}_{\mathbf{0}} \simeq 100$ at a few $\mathrm{GeV}$, which is the energy range relevant for radio synchrotron emission. This value is consistent with the predictions from Fermi shock acceleration and hadronic interaction models (see Table 1). At lower and higher energies, however, $\mathbf{K}$ may vary with particle energy (see Sect. 3.4.

The observed energy spectrum of cosmic rays is the result of balance between injection, propagation and energy losses. Interactions with matter and radiation are different for protons and electrons, so that the shape of their energy spectra are generally different (Pohl 1993a, Lieu et al. 1999 Schlickeiser 2002). At low energies (typically below a few $100 \mathrm{MeV}$ ) the dominating loss of cosmic ray protons and electrons is ionization of the neutral gas and/or Coulomb interaction with the ionized gas. At energies beyond $1 \mathrm{GeV}$ the dominating loss of protons (and other nucleons) are inelastic collisions with atoms and molecules, producing pions and secondary electrons. The spectral index of the stationary energy spectrum is not changed. The dominating loss mechanism for electrons is nonthermal bremsstrahlung producing low-energy $\gamma$-rays (Schlickeiser2002 p. 100). At even higher energies (beyond a few $\mathrm{GeV}$ ) the electrons suffer from synchrotron and inverse Compton losses (see Sect. 3.4). Furthermore, the spectra of all cosmic ray species in galaxies may be steepened if particle diffusion (escape) is energydependent. As the result of energy loss processes, the cosmic ray electron spectrum is not proportional to the proton spectrum, so that $\mathbf{K}$ varies with energy. Only in a relativistic electron/positron plasma as discussed for jets and lobes of radio galaxies, where cosmic ray protons are absent, $\mathcal{K}=0$ and $\mathbf{K}=0$ are valid at all energies (see Sects. 3.3 and 4.3).

In this paper we present an easily applicable formula with two input parameters from observations, synchrotron intensity and spectral index. We discuss the energy/frequency range where the revised formula can be applied because a reliable and constant value for the proton-electron number density ratio $\mathbf{K}_{\mathbf{0}}$ can be adopted. As a result, a more accurate estimate of the equipartition field strength is possible than in the classical approach.

Pfrommer \& Enßlin (2004b) give two formulae, for the classical minimum-energy criterion and for the hadronic interaction model applied to two galaxy clusters, taking into account (2) and (3) discussed above. Their formula (6.2) for the classical case includes luminosity, cluster volume, the protonto-electron energy density ratio and the lower cut-off energy of the electron spectrum.

\section{The revised formula}

The equipartition and minimum-energy procedures need the total energy of cosmic rays, to be derived from the observed 
radio synchrotron spectrum. An accurate treatment needs to account for all energy loss processes which affect the energy spectrum of nucleons and electrons, as discussed in detail by Pohl (1993a). This method, however, requires additional knowledge of the distributions of gas density and radiation field and is applicable only to a few well-studied objects.

In this paper we derive a revised formula for the simple case that the number density ratio $\mathbf{K}_{\mathbf{0}}$ of protons and electrons is constant which is valid in a limited range of particle energies. We further assume that the cosmic rays are accelerated by electromagnetic processes which generate power laws in momentum, and that the same total numbers of protons and electrons are accelerated. The total energy is dominated by the protons. As the proton energy spectrum flattens below the fixed proton rest energy $E_{\mathrm{p}}$, the total cosmic ray energy can be computed easily. The details are presented in the Appendix.

\subsection{Revised equipartition formula for the total field}

From the total cosmic ray energy density $\epsilon_{\mathrm{CR}}$ as a function of field strength $B$ (Eq. A16, see Appendix) and assuming $\epsilon_{\mathrm{CR}}=\epsilon_{\mathrm{B}}=B_{\text {eq }}^{2} / 8 \pi$ we get:

$$
\begin{aligned}
B_{\mathrm{eq}}= & \left\{4 \pi(2 \alpha+1)\left(\mathbf{K}_{\mathbf{0}}+1\right) I_{\nu} E_{\mathrm{p}}^{1-2 \alpha}\left(\nu / 2 c_{1}\right)^{\alpha}\right. \\
& \left./\left[(2 \alpha-1) c_{2}(\alpha) l c_{4}(i)\right]\right\}^{1 /(\alpha+3)}
\end{aligned}
$$

$I_{\nu}$ is the synchrotron intensity at frequency $\nu$ and $\alpha$ the synchrotron spectral index. $\mathbf{K}_{\mathbf{0}}$ is the constant ratio of the number densities of protons and electrons in the energy range where their spectra are proportional (see Sect. 3.4). $E_{\mathrm{p}}$ is the proton rest energy. $c_{1}, c_{2}$ and $c_{4}$ are constants. $c_{2}$ and $c_{4}$ depend on $\alpha$ and the magnetic field's inclination, respectively (see the Appendix for details).

$I_{\nu}$ and $\alpha$ can be determined from observations, while the proton-to-electron ratio $\mathbf{K}_{\mathbf{0}}$ and pathlength $l$ have to be assumed. If the synchrotron sources have a volume filling factor $f, l$ has to be replaced by $l \times f$ in order to obtain $B_{\text {eq }}$ within the source. $B_{\text {eq }}$ depends only weakly on the source's distance via the dependence on $l$.

We have restricted the discussion in this paper to nearby sources. For redshifts $z>0$, correction terms are required which are given e.g. in Govoni \& Feretti (2004).

Eq. (3) yields field strengths which are larger by $7 \%$ for $\alpha=0.6$, larger by $2 \%$ for $\alpha=0.75$, and smaller by $8 \%$ for $\alpha=1.0$ compared to the results obtained with the earlier version of the revised equipartition formula (e.g. Krause et al. 1984, Beck 1991, Niklas 1997, Thierbach et al. 2003). The reason is that a simplified version of Eq. A12 was used previously, with integration only from $E_{1}=300 \mathrm{MeV}$ to $E_{2} \rightarrow \infty$. However, the differences to Eq. (3) are small, smaller than the typical errors caused by uncertainties in the input values, so that the values published by the Bonn group previously are still valid.

In case of equilibrium between magnetic and cosmic ray pressures, the field estimate (Eq. (3) has to be reduced by the factor $3^{-1 /(\alpha+3)}$.
A formula similar to Eq. (3) has been derived by Brunetti et al. (1997). However, Eq. (A3) in Brunetti et al., which includes the lower energy cutoff of the cosmic ray electron spectrum, is not applicable in the case of dominating protons.

\subsection{Revised minimum energy formula for the total field}

From Eq. A16 (Appendix) and $d \epsilon_{\text {tot }} / d B=0$ we get:

$$
\begin{aligned}
B_{\min }= & \left\{2 \pi(2 \alpha+1)(\alpha+1)\left(\mathbf{K}_{\mathbf{0}}+1\right) I_{\nu} E_{\mathrm{p}}^{1-2 \alpha}\right. \\
& \left.\times\left(\nu / 2 c_{1}\right)^{\alpha} /\left[(2 \alpha-1) c_{2}(\gamma) l c_{4}(i)\right]\right\}^{1 /(\alpha+3)}
\end{aligned}
$$

The ratio of minimum-energy magnetic and cosmic ray energy densities is:

$\epsilon_{\mathrm{B}} / \epsilon_{\mathrm{CR}}=(\alpha+1) / 2$

Hence, the ratio of minimum-energy (4) and equipartition (3) field strengths is not constant, as in the classical case (1), but depends on the synchrotron spectral index $\alpha$ :

$B_{\min } / B_{\text {eq }}=[(\alpha+1) / 2]^{1 /(\alpha+3)}$

For $\alpha=1$ the revised formula gives identical results for the equipartition and minimum energy conditions.

The revised formula is not valid for $\alpha \leq 0.5(\gamma \leq 2)$ because the second integral in Eqs. A5 and A12 diverges for $E_{2} \rightarrow \infty$. Such flat injection spectra are observed in a few young supernova remnants and can be explained by Fermi acceleration operating in a strongly magnetized (low$\beta$ ) plasma (Schlickeiser \& Fürst 1989). In the diffuse medium of galaxies, radio lobes and clusters, $\alpha \leq 0.5$ is observed only at low frequencies and indicates strong energy losses of the electrons due to ionization and/or Coulomb interaction (Pohl 1993a, Sarazin 1999) where the formula cannot be used.

Strong shocks in a non-relativistic $\beta \geq 1$ plasma may generate injection spectra with $\alpha=0.5$ (Schlickeiser \& Fürst 1989) where the total cosmic ray energy is computed according to Eq. A14 and the minimum energy formula has to be modified accordingly:

$$
\begin{aligned}
B_{\min }= & \left\{4 \pi(\alpha+1)\left(\mathbf{K}_{\mathbf{0}}+1\right) I_{\nu} E_{0}^{1-2 \alpha}\left[\frac{1}{2}+\ln \left(E_{2} / E_{\mathrm{p}}\right)\right]\right. \\
& \left.\times\left(\nu / 2 c_{1}\right)^{\alpha} /\left[c_{2}(\gamma) l c_{4}(i)\right]\right\}^{1 /(\alpha+3)}
\end{aligned}
$$

Fig. 11 shows the ratio $q$ between the revised minimumenergy field strength $B_{\min }$ (Eq. (5)) and the classical value $B_{\text {class }}$ (Eq. A20), using ratios of $\mathbf{K}_{\mathbf{0}}=100$ and $\mathcal{K}=100$, respectively. For $\alpha \simeq 0.5(\gamma \simeq 2)$ the ratio is almost constant and about 2. For larger values of $\alpha$, the ratio becomes a function of the field strength $B_{\mathrm{min}}$. For weak fields (below a few $\mu \mathrm{G}$ ) and values of $\alpha$ between $\simeq 0.6$ and $\simeq 1$ the revised value differs insignificantly (with respect to the typical errors of $20 \%-30 \%$ ) from the classical one.

For flat radio spectra $(0.5<\alpha<0.6)$ the classical estimate is too low because the fixed upper limit $\nu_{\max }=10 \mathrm{GHz}$ used for integrating the electron energy excludes the highenergy part of the cosmic ray spectrum carrying a significant fraction of the total energy. On the other hand, the classical 


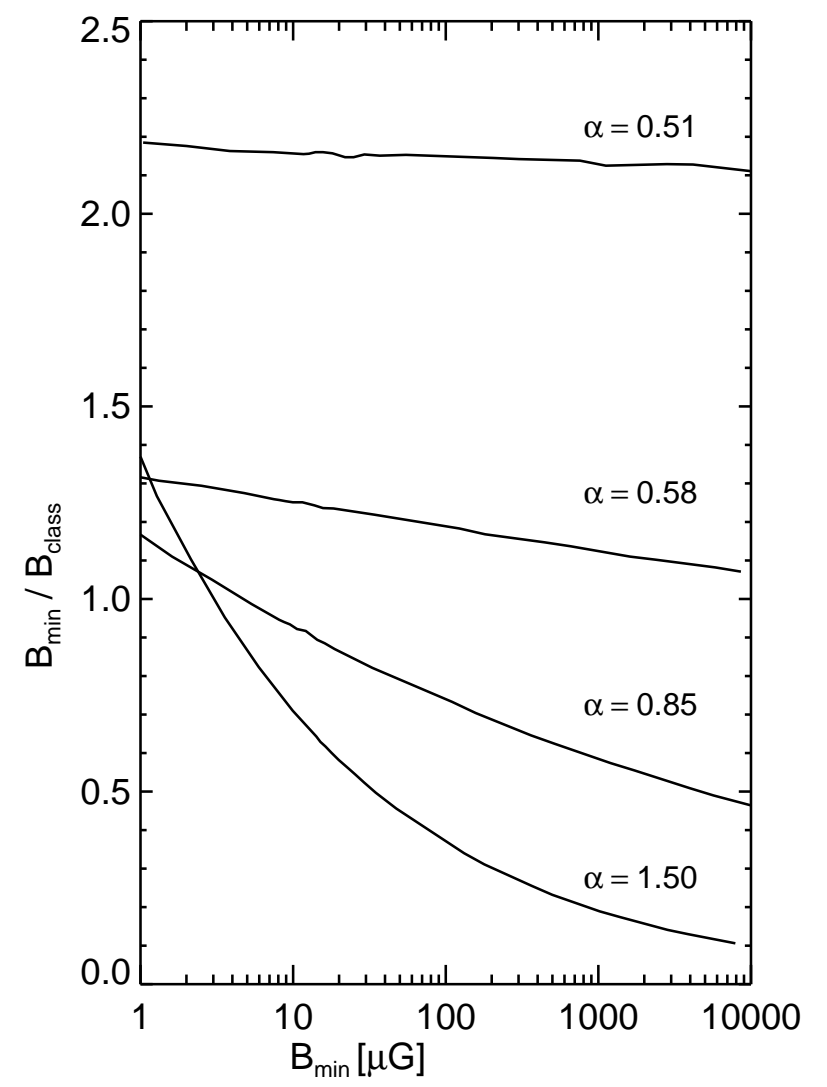

Fig. 1. Ratio between the revised minimum-energy field strength $B_{\min }$ (Eq. (4)) and the classical value $B_{\text {class }}$ (Eq. A20, assuming $\nu_{\min }=10 \mathrm{MHz}$ and $\nu_{\max }=10 \mathrm{GHz}$ ) as a function of the revised field strength $B_{\min }$ and the synchrotron spectral index $\alpha$. A proton-to-electron number density ratio of $\mathbf{K}_{\mathbf{0}}=100$ was adopted.

estimate is too high for steep radio spectra $(\alpha>0.7)$ if the field strength is $>10 \mu \mathrm{G}$. Here the lower integration limit $\nu_{\min }=10 \mathrm{MHz}$ corresponds to energies $<250 \mathrm{MeV}$ which is below the lower break energy $E_{\mathrm{p}}=938 \mathrm{MeV}$ of the proton spectrum (see Sect. 3.4, , so that the total cosmic ray energy is overestimated.

The ratio $B_{\min } / B_{\text {class }}$ depends weakly on $\mathbf{K}_{\mathbf{0}}(q \propto$ $\mathbf{K}_{\mathbf{0}}{ }^{(1 /(\alpha+3))-2 / 7}$ ) and on the frequency limits used for the classical formula. For example, $q$ is about $10 \%$ smaller for $\alpha=0.51$ when increasing $\nu_{\max }$ from $10 \mathrm{GHz}$ to $100 \mathrm{GHz}$.

\subsection{Relativistic electron/positron pair plasma}

Jets of radio galaxies may be composed of electrons and positrons (Bicknell et al.2001). Shock acceleration acting on a relativistic electron/positron pair plasma generates a power law in momentum which leads to a break in the energy spectrum at $E_{\mathrm{e}}=511.00 \mathrm{keV}=8.1871 \cdot 10^{-7} \mathrm{erg}$, but this break is not observable in the radio range because low-frequency radio spectra are flattened by ionization and/or Coulomb losses causing a break at $E_{\mathrm{i}}\left(E_{\mathrm{i}}>E_{\mathrm{e}}\right)$ in the stationary energy spectrum (see Sect. 3.4). The revised formulae (3) or (4) can be applied to an electron/positron plasma by using $\mathbf{K}_{\mathbf{0}}=0$ and replacing the lower energy break $E_{\mathrm{p}}$ by $E_{\mathrm{i}}$. If, however, synchrotron or inverse Compton loss is significant beyond $E_{\mathrm{syn}}$, the total cosmic ray energy as to be computed by integration over the observed spectrum, not according to Eq. A16.

The ratio of the revised field strength in a relativistic electron/positron plasma $B_{\min , \mathrm{e}}\left(\mathbf{K}_{\mathbf{0}}=0\right)$ to that in a proton-dominated plasma $B_{\min }$ (using Eq. (7) and assuming $\left.\left(\mathbf{K}_{\mathbf{0}}+1\right) \simeq \mathbf{K}_{\mathbf{0}}\right)$ is:

$$
\begin{aligned}
B_{\min , \mathrm{e}} / B_{\min }= & \left(E_{\mathrm{p}} / E_{\mathrm{e}}\right)^{-\alpha_{0} /(\alpha+3)} \\
& \times\left(E_{\mathrm{p}} / E_{\mathrm{i}}\right)^{(2 \alpha-1) /(\alpha+3)}
\end{aligned}
$$

where $\alpha_{0}$ is the spectral index of the injection synchrotron spectrum. $B_{\min , \mathrm{e}} / B_{\min }$ varies only weakly with spectral index $\alpha$ and lower energy break $E_{\mathrm{i}}$. For a wide range in $\alpha_{0}$ and $\alpha(0.5-0.75)$ and in $E_{\mathrm{i}}(10-100 \mathrm{MeV}), B_{\min , \mathrm{e}} / B_{\min } \simeq 1 / 3$.

\subsection{Proton-to-electron ratio and energy losses}

The ratio $\mathbf{K}(\mathrm{E})$ of the proton-to-electron number densities per particle energy interval $\left(n_{\mathrm{p}} / n_{\mathrm{e}}\right)$ depends on the acceleration process, the propagation and the energy losses of protons and electrons. In the range of particle energies $E_{\mathrm{p}}<E<E_{\text {syn }}$, where losses are small or affect protons and electrons in the same way ("thin target", see below), $\mathbf{K}=\mathbf{K}_{\mathbf{0}}$ is constant.

For electromagnetic acceleration mechanisms which generate a power law in momentum, $\mathbf{K}_{\mathbf{0}}$ depends only on the transition energies from the non-relativistic to the relativistic regime for protons and electrons, $E_{\mathrm{p}}$ and $E_{\mathrm{e}}$, and on the particle injection spectral index $\gamma_{0}$ (Eq. A4); see also Bell 1978, Schlickeiser 2002 p. 472):

$$
\begin{aligned}
\mathbf{K}_{\mathbf{0}} & =n_{\mathrm{p}, 0} / n_{\mathrm{e}, 0}=\left(E_{\mathrm{p}} / E_{\mathrm{e}}\right)^{\left(\gamma_{0}-1\right) / 2} \\
& =\left(E_{\mathrm{p}} / E_{\mathrm{e}}\right)^{\alpha_{0}} \quad\left(E_{\mathrm{p}}<E<E_{\mathrm{syn}}, \text { thin target }\right)
\end{aligned}
$$

where $\gamma_{0}$ is the spectral index of the injection cosmic ray spectrum. $\gamma_{0}$ is related to the shock compression ratio $r$ via $\gamma_{0}=(r+2) /(r-1)$ (see Appendix). For $\gamma_{0} \simeq 2.2$, as expected from acceleration in supernova remnants, we get $\mathbf{K}_{\mathbf{0}} \simeq 100$, consistent with the local Galactic cosmic ray data near the sun at particle energies of a few GeV (see Appendix).

Eqs. A6 and (7) are used to compute the intrinsic ratio $\mathcal{K}$ of total energies of protons and electrons (for negligible energy losses):

$\mathcal{K}=\left(E_{\mathrm{p}} / E_{\mathrm{e}}\right)^{\left(3-\gamma_{0}\right) / 2}$

Fig.2] shows the variation of $\mathbf{K}_{\mathbf{0}}$ and $\mathcal{K}$ with the compression ratio $r$ in the shock. Table 1 gives values of $\mathbf{K}_{\mathbf{0}}$ and $\mathcal{K}$ for various injection processes. For strong shocks in non-relativistic gas $\left(r=4, \gamma_{0}=2\right) \mathcal{K}=\mathbf{K}_{\mathbf{0}} \simeq 40$. For steeper intrinsic spectra (weaker shocks) $\mathcal{K}$ decreases, while $\mathbf{K}_{\mathbf{0}}$ increases (Fig. 2). This demonstrates why the classical formula using $\mathcal{K}$ is of little practical use: The number density ratio $\mathbf{K}_{\mathbf{0}}$, in the particle energy range relevant for the observed synchrotron intensity, is not proportional to the energy ratio $\mathcal{K}$.

In galaxy clusters secondary electrons are generated by hadronic interactions between cosmic ray protons and nuclei of the hot gas and may contribute to synchrotron emission 
Table 1. Cosmic-ray proton/electron number density ratio $\mathbf{K}_{\mathbf{0}}$ ("thin target") and the ratio $\mathcal{K}$ of total energy densities for various injection mechanisms

\begin{tabular}{lll}
\hline CR origin & $\mathbf{K}_{\mathbf{0}}$ & $\mathcal{K}$ \\
\hline Fermi shock acceleration (strong shocks, non-relativistic gas) & $40-100$ & $40-20$ \\
Secondary electrons & $100-300$ & $\gg 1$ \\
Turbulence (resonant scattering of Alfvén waves) & $\simeq 100$ & $\simeq 20$ \\
Pair plasma & 0 & 0 \\
\hline
\end{tabular}

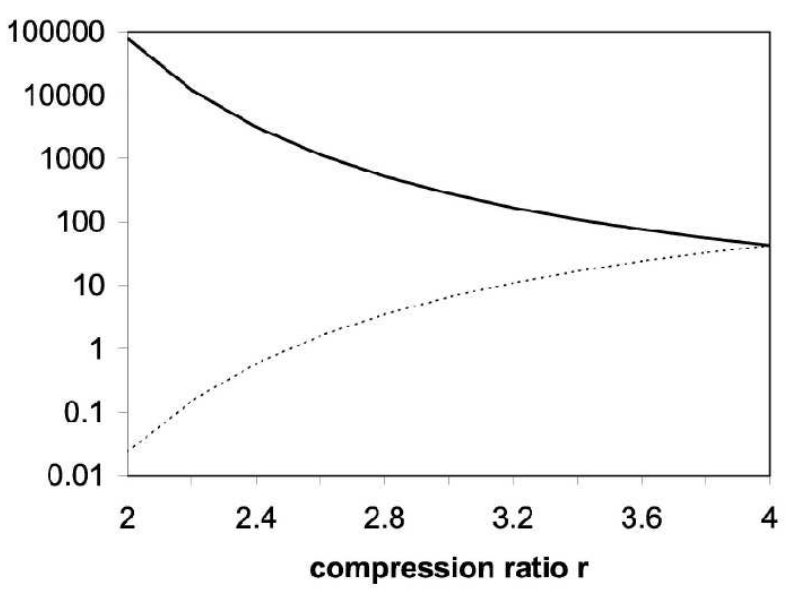

Fig. 2. Variation of the intrinsic ratio of proton-to-electron number densities $\mathbf{K}_{\mathbf{0}}$ (thick line) and of the intrinsic ratio of total energy densities $\mathcal{K}$ (dotted line) with the shock compression ratio $r$. Energy losses are assumed to be negligible.

(Dennison 1980). The spectral index $\gamma_{\mathrm{e}}$ of the electron spectrum is larger (steeper) than that of the protons $\gamma_{p}$, depending on the model (Pfrommer \& Enßlin 2004a). As only a small fraction of the proton energy can be converted into secondary electrons, the ratio of total proton/electron energies is $\mathcal{K} \gg 1$. Assuming balance between injection and losses, Dennison (1980) estimated $\mathcal{K}=5\left[9+(B / \mu G)^{2}\right]$. Typical expected values for the number density ratio in galaxy clusters are $\mathbf{K}_{\mathbf{0}}=100-300$ above particle energies of a few $\mathrm{GeV}$ (Pfrommer \& Enßlin 2004b).

Eq. (7) is valid (i.e. $\mathbf{K}_{\mathbf{0}}$ is constant) in the energy range $E_{\mathrm{p}}<E<E_{\mathrm{syn}}$ if the spectral indices of the electrons and protons are the same. This can be achieved if energy losses are negligible, or if the timescale for cosmic ray escape loss is smaller than that for electron loss by nonthermal bremsstrahlung, which is the case for low interstellar or intergalactic gas densities ("thin target", Pohl 1993a), so that nonthermal bremsstrahlung is unimportant for the stationary electron spectrum. Energy-dependent escape steepens all cosmic ray spectra in the same way, $\gamma_{\mathrm{e}}=\gamma_{\mathrm{p}}=\gamma_{0}+\Delta \gamma$, where $-\Delta \gamma \simeq-0.6$ is the exponent of the energy-dependent escape time $t_{\text {esc }}$ (see Appendix), and the revised formula for equipartition or minimum energy can be applied.

If, however, the gas density is high or the escape time is large, the object is a "thick target" for electrons, so that nonthermal bremsstrahlung loss dominates the electron spectrum at $\mathrm{GeV}$ energies. The slopes of the electron and the radio synchrotron spectra are the same as for the injection spectra $\left(\gamma_{0}\right.$ and $\alpha_{0}$, respectively). As the loss rate increases linearly with gas density $n$, the proton-to-electron ratio increases with gas density. If the proton spectrum beyond $E_{\mathrm{p}}$ is steepened by energy-dependent escape $\left(t_{e s c} \propto E^{-\Delta \gamma}\right)$, e.g. in galaxies, the ratio $\mathbf{K}(\mathrm{E})$ also depends on energy:

$\mathbf{K}(E) \propto n E^{-\Delta \gamma} \quad\left(E_{\mathrm{p}}<E<E_{\text {syn }}\right.$, thick target $)$

The result is that the ratio $\mathbf{K}(\mathrm{E})$ varies over the whole range of observable energies, so that the revised formula for energy equipartition or minimum energy should not be applied. The classical formula faces a similar problem as both the synchrotron intensity and the total energy ratio are affected by bremsstrahlung loss, but the effect on the ratio $\mathcal{K}$ of total energies is hardly predictable.

We will now discuss the ratio $\mathbf{K}(\mathrm{E})$ for low and high electron energies (outside the energy range $E_{\mathrm{p}}<E<E_{\mathrm{syn}}$ ). In a proton-dominated $\left(\mathbf{K}_{\mathbf{0}} \gg 1\right)$ cosmic ray plasma, the lower energy limit in Eq. (7) or (9) is set by the proton spectrum which flattens below $E_{\mathrm{p}}=938 \mathrm{MeV}$, while the electron spectrum flattens below $E_{\mathrm{e}}=511 \mathrm{keV}$ (Eq. A22). For $E<E_{\mathrm{p}}$ the proton and electron spectra are not proportional, so that $\mathbf{K}(\mathrm{E})$ is not constant:

$\mathbf{K}(E)=\left(E / E_{\mathrm{e}}\right)^{\alpha_{0}} \quad\left(E_{\mathrm{e}}<E<E_{\mathrm{p}}\right)$

The revised formula should not be applied for $E<E_{\mathrm{p}}$. In a $10 \mu \mathrm{G}$ magnetic field, electrons with $E<E_{\mathrm{p}}$ are observed at frequencies below about $140 \mathrm{MHz}$ (Eq. (2)).

The classical formula is also affected if radio observations at low frequencies are used. Even in a weak $1 \mu \mathrm{G}$ magnetic field, the minimum allowed frequency corresponding to $E_{\mathrm{p}}$ is still higher than the standard lower frequency limit of $10 \mathrm{MHz}$ used in the classical formula (Eq. (1)). The standard integration range of $\geq 10 \mathrm{MHz}$ would trace an appropriate part of the energy spectrum only if the field strength is below $0.7 \mu \mathrm{G}$. However, synchrotron emission from such weak fields is too faint to be detected with present-day radio telescopes. Hence, the lower frequency limit of $10 \mathrm{MHz}$ used in the classical formula (Eq. (1) is generally too low and leads to an overestimate of the field strength. This bias increases with increasing field strength (see Fig. 11). The ratio $\mathcal{K}$ of total energies cannot be applied to low-frequency radio data.

At low energies, energy losses modify the proton and electron spectra (Pohl 1993a) and the effective K(E) is different from that according to Eq. (10). The break in the electron spectrum by $\Delta \gamma_{\mathrm{e}}=-1$ (flattening) due to ionization and/or Coulomb loss depends on gas density (see Eq. (1) in Brunetti et al. 1997) and occurs at a few $100 \mathrm{MeV}$ in the ISM of galaxies and at a few tens $\mathrm{MeV}$ in radio lobes and clusters. These 
energies correspond to radio frequencies which are generally below the observable range, so that this effect is irrelevant for equipartition estimates in a proton-dominated relativistic plasma.

$E_{\text {syn }}$ in Eq. (7) is the upper energy break where synchrotron or inverse Compton loss of the electrons becomes dominant. Inverse Compton loss has the same energy and frequency dependence as synchrotron loss; both are of similar importance if the energy density of the radiation field and the magnetic energy density are comparable. Inverse Compton loss from the CMB background dominates if the field strength is below $3.25 \times(1+z)^{2} \mu \mathrm{G}$. For $E_{\mathrm{e}}>E_{\mathrm{syn}}$ the spectral index steepens by $\Delta \gamma_{\mathrm{e}}=1$, observable as a smooth steepening of the synchrotron spectrum by $\Delta \alpha=0.5$. In the revised formula for energy equipartition or minimum energy one should not use data at radio frequencies corresponding to the electron energy range $E>E_{\mathrm{syn}}$, where their energy spectrum is not proportional to the proton spectrum and the ratio $\mathbf{K}$ is a function increasing with $E$ (Pohl 1993a, Lieu et al. 1999). A simplified estimate is:

$\mathbf{K}(E)=\mathbf{K}_{\mathbf{0}}\left(E / E_{\mathrm{syn}}\right) \quad\left(E>E_{\mathrm{syn}}\right)$

Using instead $\mathbf{K}_{\mathbf{0}}$ in the revised formula leads to an underestimate of the field strength. Again, the classical formula has a similar problem because the ratio $\mathcal{K}$ of total energies also increases in the case of strong synchrotron or inverse Compton loss.

If the field strength varies along the line of sight or within the volume observed by the telescope beam, synchrotron loss may lead to an anticorrelation between field strength and cosmic ray electron density, so that the equipartition field is underestimated further (Beck et al. 2003).

Synchrotron loss is significant in sources with strong magnetic fields like young supernova remnants, starburst galaxies and radio lobes (Sects. 4.2 and 4.3, and also in galaxies away from the acceleration sites of cosmic rays, for example in interarm regions, in the outer disk and in the halo (Sect.4.1).

The various bias effects are summarized in Table 2

\subsection{Equipartition and minimum-energy estimates of the regular field}

Knowing the equipartition or minimum-energy estimate of the total field strength, the equipartition or minimum-energy estimate of the regular field strength $B_{\mathrm{reg}, \perp}$ in the sky plane can be computed from the observed degree of polarization $p$ of the synchrotron emission:

$p=p_{0}\left(1+\frac{7}{3} q^{2}\right) /\left(1+3 q^{2}+\frac{10}{9} q^{4}\right)$

where $p_{0}$ is the intrinsic degree of polarization $\left(p_{0}=(3-\right.$ $3 \alpha) /(5-3 \alpha)$ ) and $q$ is the ratio of the isotropic turbulent field $B_{\text {turb }}$ to the regular field component $B_{\text {reg, }, \perp}$ in the sky plane (Beck et al.2003). For the case of a dominating turbulent field $(q \gg 1), p \simeq 2.1 p_{0} q^{-2}$.

\section{Discussion and examples}

\subsection{Weak fields: spiral galaxies}

The Milky Way and most spiral galaxies have steep radio spectra in the frequency range of a few $\mathrm{GHz}$ which indicates that the energy spectra of their cosmic ray electrons are steepened by escape loss (see Appendix). Galaxies are "thin targets" for cosmic ray electrons, except for massive spiral arms and starburst regions (see Sect.4.2). The revised formula can be applied, using the part of the radio spectrum below the break frequency $\nu_{\text {syn }}$ beyond which synchrotron or inverse Compton losses become important. The upper energy limit $E_{\text {syn }}$ corresponding to $\nu_{\text {syn }}$ can be estimated as follows.

The synchrotron lifetime $t_{\text {syn }}$ for electrons of energy $E$ is (Lang 1999 p. 32):

$$
\begin{aligned}
t_{\mathrm{syn}} & =8.35 \cdot 10^{9} \mathrm{yr} /\left[\left(B_{\perp} / \mu \mathrm{G}\right)^{2}\left(E_{\mathrm{syn}} / \mathrm{GeV}\right)\right] \\
& =1.06 \cdot 10^{9} \mathrm{yr} /\left[\left(B_{\perp} / \mu \mathrm{G}\right)^{1.5}\left(\nu_{\mathrm{syn}} / \mathrm{GHz}\right)^{0.5}\right]
\end{aligned}
$$

Note that the constant is different from that used in most papers (e.g. Carilli et al. 1991, Mack et al. 1998, Feretti et al. 1998). The escape time $t_{\text {esc }}$ in the Milky Way is $\simeq 10^{7} \mathrm{yr}$ at non-relativistic and mildly relativistic energies (Engelmann et al. 1990, Schlickeiser 2002, p. 439), so that the break in the radio frequency spectrum is expected at:

$\nu_{\mathrm{syn}} \approx 10 \mathrm{GHz} /\left(B_{\perp} / 10 \mu \mathrm{G}\right)^{3}$

For the typical strength of ISM magnetic fields of $10 \mu \mathrm{G}$ (Niklas 1995) we get $\nu_{\text {syn }} \simeq 10 \mathrm{GHz}$. At higher frequencies the synchrotron intensity $I_{\nu}$ and the spectral index $\alpha$ can be used neither for the revised formula nor for the classical formula. If the resulting field strength is larger than $10 \mu \mathrm{G}$, like in grand-design spiral galaxies, the useful frequency range shifts to even lower frequencies. Furthermore, the thermal contribution to the total radio intensity increases with increasing frequency and has to be subtracted.

In galaxies with a high star-formation rate, galactic winds may form. The escape time may then be shorter than in the Milky Way and $\nu_{\text {syn }}$ is larger than according to Eq. (14). Signature of galactic winds is that at low frequencies the local radio spectrum in edge-on galaxies remains flat up to large heights above the disk (Lerche \& Schlickeiser 1982). In the Milky Way, the radio spectral index distribution is consistent with a galactic wind (Reich \& Reich 1988).

Pohl (1993a) argued that in the spiral galaxy M 51 the spectral index of the total radio emission (integrated over the whole galaxy) is $\alpha \simeq 0.5$ ( $\gamma_{0} \simeq 2.0$ ) at low frequencies, but a frequency break at about $1.4 \mathrm{GHz}$ due to energy losses causes a steepening of almost the whole observable radio spectrum. As a result, the classical minimum-energy field strength in the spiral galaxy M 51 is too high. However, the compilation of all data by Niklas (1995) does not indicate a significant steepening of the radio spectrum of M 51 until $25 \mathrm{GHz}$, but a flattening due to thermal emission. In his sample of spiral galaxies, Niklas (1995) found only very few cases of spectral steepening. Hence, escape seems to be the dominant loss process in the spectra of integrated radio emission of galaxies 
Table 2. Bias of equipartition field estimates. $\mathbf{K}(\mathrm{E})$ is the cosmic ray proton/electron ratio, where $\mathbf{K}_{\mathbf{0}}$ denotes a value which does not vary with energy

\begin{tabular}{|c|c|c|c|c|}
\hline Method & Cosmic-ray composition & Bias effect & Field strength & Reference \\
\hline Classical & $p^{+}+e^{-}\left(\mathbf{K}_{\mathbf{0}} \simeq 100\right)$ & Integration over frequency & $\begin{array}{l}\text { Underestimate }(\alpha<0.6) \\
\text { Overestimate }(\alpha>0.7)\end{array}$ & $\begin{array}{l}\text { This paper } \\
\text { (Fig.1 }\end{array}$ \\
\hline Classical & $e^{-}+e^{+}\left(\mathbf{K}_{\mathbf{0}}=0\right)$ & $\begin{array}{l}\text { Fixed frequency range } \\
\text { (e.g. } 10 \mathrm{MHz}-10 \mathrm{GHz} \text { ) }\end{array}$ & $\begin{array}{l}\text { Underestimate (weak fields) } \\
\text { Overestimate (strong fields) }\end{array}$ & This paper \\
\hline Classical+revised & $p^{+}+e^{-}(\mathbf{K}(\mathrm{E})>100)$ & $\begin{array}{l}\text { Synchrotron/IC/ } \\
\text { bremsstrahlung losses }\end{array}$ & Underestimate & This paper \\
\hline Classical+revised & any & $\begin{array}{l}\text { Field fluctuations without/ } \\
\text { with synchrotron loss }\end{array}$ & $\begin{array}{l}\text { Overestimate (weak fields)/ } \\
\text { Underestimate (strong fields) }\end{array}$ & Beck et al.2003 \\
\hline
\end{tabular}

between about $100 \mathrm{MHz}$ and $10 \mathrm{GHz}$. (This does not hold for spectra of the local emission, see below.)

Fitt \& Alexander (1993) used the $1.49 \mathrm{GHz}$ radio fluxes of 146 spiral and irregular galaxies to derive minimumenergy field strengths with the classical formula, simply assuming a constant spectral index of $\alpha=0.75$, a negligible thermal fraction and the standard frequency limits. Their distribution peaks around $11 \mu \mathrm{G}$ with a standard deviation $\sigma$ of $4 \mu \mathrm{G}$ (for a ratio of total energies $\mathcal{K}$ of 100).

Niklas (1995) observed a sample of spiral galaxies in radio continuum at $10.55 \mathrm{GHz}$ and compiled all available data at other frequencies. Based on the spectra of the integrated radio emission, Niklas et al. (1997) could separate the synchrotron from the thermal emission for 74 galaxies. The mean thermal fraction at $1 \mathrm{GHz}$ is $8 \%$, and the mean synchrotron spectral index is $\alpha=0.83$ with a standard deviation of 0.13 . Hence, the average spectrum of the extragalactic cosmic ray electrons (and probably also that of the protons) at particle energies of a few $\mathrm{GeV}$ has the same spectral index $(\gamma \simeq 2.7)$ as that in the Milky Way. Niklas (1995) derived galaxyaveraged field strengths according to the classical formula A20 and to a revised formula similar to Eq. (4), approximating integration $\mathrm{A} 12$ by one integral from $E_{1}=300 \mathrm{MeV}$ to infinity. His results (for $\mathbf{K}_{\mathbf{0}}=100$ ) are similar for the two cases, as expected from Fig. 11 The mean is $9 \mu \mathrm{G}$ (with a standard deviation $\sigma=3 \mu \mathrm{G}$ ) both for the revised formula and for the classical case. The distribution of field strengths is more symmetrical for the revised case.

However, application of the equipartition formula to regions within spiral galaxies or in their radio halos needs special care. For example, the "ring" of M 31 emitting in radio continuum is formed by cosmic ray electrons, while the magnetic field extends to regions inside and outside the ring, as indicated by Faraday rotation data (Han et al. 1998). Leaving the star-formation regions, cosmic ray electrons rapidly lose their energy due to synchrotron loss. Hence, $\mathbf{K}(\mathrm{E})>100$, so that the equipartition formula underestimates the field strength inside and outside the ring. The same argument holds for the outer disk and the halo of galaxies, far away from the acceleration sites of cosmic rays.

In galaxies with strong magnetic fields like M 51, synchrotron loss may dominate already at a few $100 \mathrm{pc}$ from the acceleration sites, e.g. in interarm regions, as indicated by spectral steepening between the spiral arms (Fletcher et al. 2005). Hence, the interarm field strengths given by Fletcher et al. (2004) are underestimates.

\subsection{Strong fields: young supernova remnants and starburst galaxies}

The classical equipartition formula overestimates the magnetic field strength in objects with proton-dominated cosmic rays and with strong fields (Fig. 1), like massive spiral arms, young supernova remnants or starburst galaxies. Here the lower frequency limit is too low and hence overestimates the total cosmic ray energy (see Sect. 3.4). In a $100 \mu \mathrm{G}$ field, for example, the energy of an electron radiating at $10 \mathrm{MHz}$ is only $80 \mathrm{MeV}$, where the corresponding proton number in any medium is strongly reduced $\left(E \ll E_{\mathrm{p}}\right)$ and the proton-toelectron number density ratio $\mathbf{K}(\mathrm{E})$ may drop to values even smaller than 1 .

Hence, many values for the field strength quoted in the literature are strong overestimates. For example, Allen \& Kronberg (1998) integrated the radio spectrum from $10 \mathrm{MHz}$ to $100 \mathrm{GHz}$ of young supernova remnants in the galaxy M82 and derived field strengths of a few $\mathrm{mG}$ for $\mathcal{K}=0$, scaling with $(\mathcal{K}+1)^{2 / 7}$. The revised formula gives values which are between 4 and $50 \times$ smaller, depending on spectral index $\alpha$. Pérez-Torres et al. (2002) derived a minimum-energy field strength in SN $1986 \mathrm{~J}(\alpha=0.69)$ of about $13 \mathrm{mG} \times(\mathcal{K}+1)^{2 / 7}$ which, according to our revised formula, is too high by about one order of magnitude. On the other hand, the equipartition strength of the flat-spectrum SN 1993J ( $\alpha=0.51)$ of $38 \mathrm{mG}$ (Chandra et al. 2004) is too low; the revised formula gives $\simeq 100 \mathrm{mG} \times(\mathcal{K}+1)^{2 / 7}$ which fits better to the field strength derived from the synchrotron break energy (by inserting the $\mathrm{SN}$ age as $t_{\text {syn }}$ in Eq. (13).

Note that the cosmic ray electron distribution in young supernova remnants is not in a stationary state, so that energy losses modify the spectrum in a different way than discussed in Sect.3.4 The observed spectrum is that of injection, possibly modified by synchrotron loss, so that the revised formula can be applied.

Hunt et al. (2004) discussed the radio emission of the blue compact dwarf galaxy SBS 0335-052. Applying the classical formula (assuming $\mathcal{K}=40$ and a frequency range of 
$10 \mathrm{MHz}-100 \mathrm{GHz}$ ) these authors obtained an equipartition field of $\simeq 0.8 \mathrm{mG}$, while the value from our revised formula is about $30 \mu \mathrm{G}$ which fits much better to other starburst galaxies like M82 (Klein et al.1988) or the Antennae (Chyży $\&$ Beck 2004) or blue compact dwarf galaxies (Deeg et al. 1993). The same discrepancy may arise in galactic nuclei with starbursts. Beck et al. (2005) derived field strengths of $\simeq 60 \mu \mathrm{G}$ in the central starburst regions of the barred galaxies NGC 1097 and NGC 1365, while the classical formula would give much larger values.

However, starburst galaxies and regions of high star formation rate in the central regions and massive spiral arms of galaxies have high gas densities and mostly flat radio spectra ( $\alpha=0.4-0.7$ ), so that they probably are "thick targets" for the cosmic ray electrons. If so, the equipartition estimate is too low, and the correct value can be computed only by constructing a model of gas density and cosmic ray propagation.

\subsection{Radio lobes and galaxy clusters}

The classical equipartition field strengths are $20-100 \mu \mathrm{G}$ in the radio lobes of strong FRII radio galaxies (Brunetti et al. 1997), 100-600 $\mu \mathrm{G}$ in hot spots (Carilli et al. 1991, Harris et al. 2000, Wilson et al. 2000, Wilson et al. 2001, Brunetti et al.2001a, Hardcastle 2001, Hardcastle et al.2001, 2002), and $0.5-1 \mu \mathrm{G}$ in the diffuse gas of galaxy clusters and relics (Feretti \& Giovannini 1996). In all these cases it was assumed that relativistic protons are absent $(\mathcal{K}=0$, "absolute minimum energy") or contribute similarly to the total energy density as the electrons $(\mathcal{K}=1)$.

According to Fig. 2$] \mathcal{K} \leq 1$ could also be the result of a weak shock. However, particle acceleration at low Mach numbers is inefficient (Bogdan \& Völk 1983, Völk et al. 1988), so that its contribution to the total cosmic ray population is negligible.

If $\mathcal{K}=0$, the radio spectrum reflects the energy spectrum of the total cosmic rays, and the equipartition formula gives reliable results, supposing that the integration limits are set properly (Sect.3.3). Integration over the range of Lorentz factors of the electrons/positrons according to the breaks as observed in the radio spectrum of sources leads, in the case of weak magnetic fields, to slightly larger field strengths than applying the classical formula with fixed frequency limits (Brunetti et al.1997). However, the lower break energy $E_{\mathrm{i}}$ in the electron/positron energy spectrum needed for the equipartition estimate may correspond to a radio frequency which is too low to be observed. For example, electrons with the minimum Lorentz factor of 50 assumed for the lobes of 3C219 (Brunetti et al. 1999) in a $10 \mu \mathrm{G}$ field radiate at $100 \mathrm{kHz}$, a frequency much below the observable range. Here the frequency limit of $\nu_{\min }=10 \mathrm{MHz}$ assumed in the classical method is too high, and hence the field strength is underestimated, by $1.5 \times$ and $2 \times$ for $\alpha=0.8$ and $\alpha=1$, respectively, or by an even larger factor if the minimum Lorentz factor is smaller than 50 .

If the fields are strong, the break energy $E_{\mathrm{i}}$ of the electron spectrum is observable in the radio spectrum. Hardcastle et al. (2002) determined typical minimum Lorentz factors of 1000 from the breaks observed at $0.5-1 \mathrm{GHz}$ in hot spots of radio lobes and estimated field strengths of 100-200 $\mu \mathrm{G}$ (assuming equipartition with $\mathbf{K}_{\mathbf{0}}=0$ ). In such cases, the classical formula gives overestimates because $\nu_{\min }=10 \mathrm{MHz}$ is too small.

The occurence of electron/positron plasmas $(\mathcal{K}=0)$ in astronomical objects is under debate. A relativistic electron/positron plasma was discussed for the jets (Bicknell et al. 2001). However, observational evidence tends to favour an electron/proton plasma (Leahy et al. 2001). Even if jets do eject electrons and positrons into a cluster with the same energy spectrum, these particles will not survive long enough to generate significant radio emission from the IGM medium, so that (re)acceleration by intergalactic shocks (Blandford \& Ostriker 1978, Sarazin 1999, Gabici \& Blasi 2003), by interaction with MHD waves (Schlickeiser \& Miller 1998. Brunetti et al. 2001b. Fujita et al. 2003), or by reconnection (Hanasz \& Lesch 2003), or production of secondary electrons (Pfrommer \& Enßlin 2004a) is necessary.

If, however, the contribution of relativistic protons to the total cosmic ray energy is dominant $(\mathcal{K} \gg 1)$, as predicted by all electromagnetic acceleration models (Table 1 , the equipartition field strength increases with respect to that for $\mathcal{K}=0$. In the hot spots of radio lobes (Wilson et al. 2001. Hardcastle 2001, Hardcastle et al.2001 2002) stronger equipartition fields would fit much better to the high field strengths derived from Faraday rotation measures (Feretti et al. 1995, Fusco-Femiano et al. 1999.

Another reason for systematic field underestimates are energy losses. The electron spectrum is a result of the balance between acceleration and energy losses. Ionization loss dominates at low energies; at high energies synchrotron loss dominates in clusters with magnetic fields stronger than a few $\mu \mathrm{G}$ while inverse Compton loss due to CMB photons dominates in clusters with weaker fields (Sarazin 1999). $E>E_{\text {syn }}$ is the energy range where synchrotron and/or inverse Compton losses are strong and the electron spectrum is steeper than the proton spectrum $\left(\gamma_{\mathrm{e}}=\gamma_{\mathrm{p}}+1\right)$. The proton-to-electron number density ratio $\mathbf{K}$ increases with energy according to Eq. (11). In this case, the revised formula underestimates the field strength. For example, using a value for $\mathbf{K}$ of 100 instead of 1000 would underestimate the equipartition field strength by a factor of about 2 . The classical formula has a similar problem.

Synchrotron and inverse Compton losses steepen the radio spectra. The radio spectra of most radio lobes and hot spots in the range $0.4-15 \mathrm{GHz}$ are well approximated by power laws with spectral indices between of $\alpha \simeq 0.7-0.8$ in the frequency range $0.4-15 \mathrm{GHz}$, with a spectral break to $\alpha>1$ between the radio and $\mathrm{X}$-ray regimes. Meisenheimer et al. (1989) fitted the spectra of five bright hot spots with a low-frequency spectral index of $\alpha \simeq 0.5$ with smooth breaks which start to steepen the spectra already beyond a few GHz. The spectral index of the diffuse radio emission from the Coma cluster is $\alpha \simeq 1.0$ below $100 \mathrm{MHz}$ and strongly steepens at higher frequencies (Fusco-Femiano et al. 1999, Thierbach et al. 2003), which is a clear sign of energy loss. The 
(revised) equipartition field strength in the Coma cluster is $0.6 \mu \mathrm{G} \times(\mathbf{K}+1)^{1 /(\alpha+3)}$ (Thierbach et al. 2003), which gives $\simeq 4 \mu \mathrm{G}$ for $\alpha=0.8$ and $\mathbf{K}=1000$. This value is not far from that derived from Faraday rotation data (Feretti et al. 1995).

Pfrommer \& Enßlin (2004b) modeled the radio spectrum of the Coma and Perseus clusters assuming equilibrium between injection of secondary electrons (hadronic interaction) and energy losses. Their self-consistent minimum-energy formula, which does not require an assumption about $\mathcal{K}$ or $\mathbf{K}$, gives slightly larger field strengths for the Coma and Perseus clusters compared with the classical formula. However, this model cannot account for the radial steepening of the radio spectra observed in both clusters.

Our revision of total field strengths may have significant effects on estimates of the age of electron populations based on the synchrotron break frequency in the radio spectrum (Eq. (13)). Application of the classical formula for objects with strong fields may significantly overestimate the field (Fig. 11 and hence underestimate the electron lifetime. More serious is the assumption of a proton/electron total energy ratio $\mathcal{K}=1$ in most papers (Carilli et al. 1991, Mack et al. 1998, Feretti et al. 1998, Parma et al. 1999) which is not supported by any acceleration model (see above). $\mathbf{K}_{\mathbf{0}} \simeq 100$ in the revised formula would increase field strength by $3.5 \times$ and hence decrease the synchrotron age by $6.5 \times$. The discrepancy between dynamical and synchrotron ages found by Parma et al. (1999) is increased. This problem needs to be re-investigated.

\subsection{Comparison with field estimates from the ratio of synchrotron to inverse Compton $\mathrm{X}$-ray intensities}

The same cosmic ray electrons emitting radio synchrotron emission also produce X-ray emission via the inverse Compton effect. The ratio of radio to X-ray intensities can be used as a measure of field strength (Harris \& Grindlay 1979. Govoni \& Feretti 2004). Comparison with the equipartition estimates reveals three cases:

(1) A high equipartition strength is needed to achieve a consistent picture.

The lobes of two low-power radio galaxies reveal an apparent deficit in X-ray emission compared with radio emission when assuming no protons $(\mathcal{K}=0$ ) (Croston et al. 2003). Relativistic protons with 300-500 times more energy than the electrons would increase the equipartition field strength from $\simeq 3 \mu \mathrm{G}$ to $\simeq 15 \mu \mathrm{G}$ and reduce the number density of electrons, which would explain the weak X-ray emission and also ensure pressure balance between the radio lobes and the external medium.

(2) A low equipartition strength is needed to achieve a consistent picture.

The equipartition values (assuming $\mathcal{K}=0$ or 1 ) are similar to those derived from X-ray emission by inverse Compton scattering of the background photons in most radio lobes (Brunetti et al. 1997) and in the Coma cluster
(Fusco-Femiano et al. 1999, see also Table 3 in Govoni \& Feretti 2004). In most hot spots the equipartition values are also similar to those derived from X-ray emission by inverse Compton scattering of the synchrotron photons ("synchrotron self-Compton emission"), e.g. in Cyg A (Harris et al. 1994, Wilson et al. 2000), 3C123 (Hardcastle et al. 2001), 3C196 (Hardcastle 2001), 3C295 (Harris et al.2000), in 3C263 and in 3C330 (Hardcastle et al. 2002). If, however, $\mathcal{K} \gg 1$, as prediced by acceleration models (Table 1 , the field estimates increase and fit better to the Faraday rotation data (see above), but contradict the X-ray data in these objects which then are "too bright" in X-rays.

(3) The equipartition strength is always too high.

Some objects are "too bright" in X-rays compared with the energy density of the cosmic ray electrons derived from the equipartition assumption with $\mathcal{K}=0$. In other words, the equipartition values even for $\mathcal{K}=0$ are already several times larger than those allowed from the ratio of radio/X-ray intensities. This is the case in the lobes of PKS 1343-601 (Tashiro et al. 1998) and 3C219 (Brunetti et al. 1999), and in the hot spots of Pictor A (Wilson et al. 2001), 3C351 (Brunetti et al. 2001a) and 3C351 (Hardcastle et al.2002). Dominating protons $(\mathcal{K} \gg 1)$ would even increase the discrepancy.

The correction introduced for strong fields by our revised equipartition formula (Fig. 1) cannot solve the problem. For example, in the bright core of the western hot spot of Pictor A, Wilson et al. (2001) found the largest discrepancy (more than 10) between the field strength derived from the classical equipartition formula $(\simeq 470 \mu \mathrm{G}$, assuming $\mathcal{K}=1)$ and that from the radio/X-ray intensity ratio $(\simeq 33 \mu \mathrm{G})$. Our revised formula (for $\mathbf{K}_{\mathbf{0}}=1$ ) reduces the equipartition value only to $\simeq 350 \mu \mathrm{G}$, not enough to remove the discrepancy. If cosmic ray protons dominate, the equipartition field strength increases to $\simeq 1 \mathrm{mG}$ (for $\mathbf{K}_{\mathbf{0}}=100$ and $\alpha=0.8$ ), so that the discrepancy between the field estimates increases further.

Carilli \& Taylor (2002) discussed possible solutions. Firstly, most of the X-ray emission may not be of inverse Compton, but of thermal origin, which should be tested with further observations. Secondly, an anisotropic pitch angle distribution of the cosmic ray electrons could weaken the synchrotron relative to the inverse Compton emission. Thirdly, in magnetic fields of about $1 \mu \mathrm{G}$ the observed synchrotron spectrum traces electrons of larger energies than those emitting the inverse Compton spectra. If the electron spectrum steepens with energy (e.g. due to energy losses), the radio emission is reduced at high energies.

Another possible (though improbable) explanation of the discrepancy is that equipartition between magnetic fields and cosmic rays is violated. An increase in electron density by typically $n_{\mathrm{e}}^{\prime} / n_{\mathrm{e}, \mathrm{eq}} \simeq 5$ is required to match the excessive $\mathrm{X}$ ray intensities in the objects listed above. The corresponding decrease in field strength for a fixed radio synchrotron intensity and $\alpha \simeq 0.8$ is $B^{\prime} / B_{\text {eq }}=\left(n_{\mathrm{e}}^{\prime} / n_{\mathrm{e}, \mathrm{eq}}\right)^{-1 /(\alpha+1)} \simeq 0.4$, and the energy density ratio $q$ between cosmic rays and magnetic fields increases by $q=\left(n_{\mathrm{e}}^{\prime} / n_{\mathrm{e}, \mathrm{eq}}\right)^{(\alpha+3) /(\alpha+1)} \simeq 30$. Such an imbalance between particle and field energies is unstable and would cause a rapid outflow of cosmic rays. 
Finally, the magnetic field may be concentrated in filaments. Intracluster magnetic fields can be amplified by shocks in merging clusters (Roettiger et al. 1999). The ratio of synchrotron to X-ray intensities from the same cosmic ray electron spectrum is $I_{\mathrm{syn}} / I_{\mathrm{X}} \propto<n_{\mathrm{e}} B^{1+\alpha}>/<n_{\mathrm{e}}>$. If small-scale fluctuations in $n_{\mathrm{e}}$ and in $B$ are uncorrelated, $I_{\text {syn }} / I_{\mathrm{X}} \propto<B^{2}>=<B>^{2} / f_{\mathrm{B}}$, where $f_{\mathrm{B}}$ is the effective volume filling factor of the magnetic field, so that the field strength estimate from the radio/X-ray intensity ratio varies with $f^{-0.5}$. To explain a $10 \times$ discrepancy between the field estimates, a filling factor of $10^{-2}$ is required. The enhanced magnetic field in the filaments leads to synchrotron loss of the electrons and hence to an anticorrelation between $n_{\mathrm{e}}$ and $B$. The $\mathrm{X}$-ray emission may be biased towards regions of weak fields, while synchrotron intensity is biased towards regions of strong fields. This explanation appears plausible and deserves further investigation.

\section{Summary}

Assuming that cosmic rays are generated by electromagnetic shock acceleration producing the same total numbers of relativistic protons and electrons with power laws in momentum, we showed that the ratio of total energies of protons and electrons $\mathcal{K}$ is about 40 for strong shocks and decreases with decreasing shock strength. The ratio $\mathbf{K}$ of number densities of protons and electrons per energy interval for particle energies $E \geq 1 \mathrm{GeV}$ is also about 40 for strong shocks and increased with decreasing shock strength. Both ratios further depend on the various energy loss processes for protons and electrons.

The classical equipartition or minimum-energy estimate of total magnetic fields strength from radio synchrotron intensities is based on the ratio $\mathcal{K}$ of the total energies of protons and electrons, a number which is hardly known because the proton and electron spectra have not been measured over the whole energy range. We propose a revised formula which instead uses the number density ratio ratio $\mathbf{K}$ in the energy interval relevant for synchrotron emission. This ratio can be adopted from the value observed in the local Galactic cosmic rays, or it can be estimated for the energy range where energy losses are negligible or particle escape loss dominates ("thin target"), so that $\mathbf{K}$ is constant $\left(\mathbf{K}_{\mathbf{0}}\right)$.

Furthermore, the classical equipartition / minimumenergy estimate is incorrect because the cosmic ray energy density is determined by integration over a fixed interval in the radio spectrum, which introduces an implicit dependence on the field strength. Applying our revised formula, the field strengths for a proton-dominated relativistic plasma are larger by up to $2 \times$ for flat radio spectra (synchrotron spectral index $\alpha<0.6$ ), but smaller by up to $10 \times$ for steep radio spectra $(\alpha>0.7)$ and for total field strengths $B>10 \mu \mathrm{G}$ (Fig.1 and Table 2). The revised formula can be applied if energy losses are negligible or if escape is the dominant loss process for cosmic ray electrons at $\mathrm{GeV}$ energies. The classical field estimates for young supernova remnants are probably too large by a factor of several. The average field strengths for spiral galaxies remain almost unchanged. For objects containing dense gas ("thick targets") where energy loss of cosmic ray electrons by nonthermal bremsstrahlung is significant, e.g. in starburst galaxies, massive spiral arms or in starburst regions in galaxies, neither the revised nor the classical estimate can be applied.

Equipartition values for radio lobes and galaxy clusters are usually computed assuming a constant cosmic ray proton/electron energy ratio of $\mathcal{K}=0$ (i.e. pure electron/positron plasma) or $\mathcal{K}=1$ (i.e. the same contribution of protons and electrons to the total cosmic ray energy). However, all current models of cosmic ray origin predict that protons are dominant $\left(\mathcal{K} \gg 1, \mathbf{K}_{\mathbf{0}} \simeq 100-300\right)$, so that the field estimate is too low by a factor $\left(\mathbf{K}_{\mathbf{0}}+1\right)^{1 /(\alpha+3)}$. Furthermore, the radio spectra of radio lobes and clusters indicate that synchrotron or inverse Compton loss of the cosmic ray electrons are significant, so that $\mathcal{K}$ and $\mathbf{K}$ and hence the field strength increase further. The revised, stronger fields in clusters are consistent with the results of Faraday rotation observations.

In case of strong fields in radio lobes, the discrepancy with the much lower field estimates from the radio/X-ray intensity ratio in several hot spots cannot be solved and requires alternative explanations, e.g. a concentration of the field in small filaments with a low volume filling factor, or a thermal origin of the X-ray emission.

Our code BFIELD to compute the strengths of the total and regular fields from total and polarized synchrotron intensities, allowing various assumptions as discussed in this paper, is available from www.mpifr-bonn.mpg.de/staff/mkrause.

Acknowledgements. We wish to thank Elly M. Berkhuijsen, Luigina Feretti, Martin Pohl, Wolfgang Reich, and Anvar Shukurov for many useful discussions, and especially Reinhard Schlickeiser for his patience to explain to us the labyrinth of cosmic ray loss processes. Leslie Hunt is acknowledged for encouraging us to publish this paper. We are grateful to our anonymous referee for his help to make the paper clearer.

\section{References}

Aharonian, F. A., et al.: 2004, Nat 432, 75

Allen, M. L., Kronberg, P. P.: 1998, ApJ 502, 218

Beck, R.: 1991, A\&A 251, 15

Beck, R.: 2000, Phil. Trans. R. Soc. London, Ser. A 358, 777

Beck, R.: 2001, Sp. Sci. Rev., 99243

Beck, R.: 2004, in From Observations to Self-consistent Modelling of the ISM in Galaxies, eds. M. A. de Avillez \& D. Breitschwerdt, Kluwer Acad. Publ., Astrophys. Sp. Sci. 289, 293

Beck, R., Brandenburg, A., Moss, D., Shukurov, A., Sokoloff, D.: 1996, ARA\&A 34, 155

Beck, R., Ehle, M., Shoutenkov, V., Shukurov, A., Sokoloff, D.: 1999, Nat 397, 324

Beck, R., Shukurov, A., Sokoloff, D., Wielebinski, R.: 2003, A\&A 411, 99

Beck, R., Fletcher, A., Shukurov, A., et al.: 2005, A\&A, submitted

Bell, A. R.: 1978, MNRAS 182, 443

Bicknell, G. V., Wagner, S. J., Groves, B. A.: 2001, in Particles and Fields in Radio Galaxies, eds. R. A. Laing \& K. M. Blundell, ASP Conf. Ser. 250, 80

Blandford, R. D.: 2001, in Particles and Fields in Radio Galaxies, eds. R. A. Laing \& K. M. Blundell, ASP Conf. Ser. 250, 487

Blandford, R. D., Ostriker, J. P.: 1978, ApJ 221, L29

Bogdan, T. J., Völk, H. J.: 1983, A\&A 122, 129

Brunetti, G., Setti, G., Comastri, A.: 1997, A\&A 325, 898 
Brunetti, G., Comastri, A., Setti, G., Feretti, L.: 1999, A\&A 342, 57 Brunetti, G., Bondi, M., Comastri, A., et al.: 2001a, ApJ 561, L157

Brunetti, G., Setti, G., Feretti, L., Giovannini, G.: 2001b, MNRAS 320,365

Burbidge, G. R.: 1956, ApJ 124, 416

Carilli, C. L., Perley, R. A., Dreher, J. W., Leahy, J. P.: 1991, ApJ 383,554

Carilli, C. L., Taylor, G. B.: 2002, ARA\&A 40, 319

Chandra, P., Ray, A., Bhatnagar, S.: 2004, ApJ 604, L97

Chi, X., Wolfendale, A. W.: 1993, Nat 362, 610

Chyży, K. T., Beck, R.: 2004, A\&A 417, 541

Croston, J. H., Hardcastle, M. J., Birkinshaw, M., Worrall, D. M.: 2003, MNRAS 346, 1041

Deeg, H.-J., Brinks, E., Duric, N., Klein, U., Skillman, E.: 1993, ApJ 410, 626

Dennison, B.: 1980, ApJ 239, L93

Drury, L. O'C.: 1983, Rep. Prog. Phys. 46, 973

Duric, N.: 1990, in IAU Symp. No. 140, in Galactic and Intergalactic Magnetic Fields, eds. R. Beck et al., Kluwer, Dordrecht, 235

Engelmann, J. J., Ferrando, P., Soutoul, A., et al.: 1990, A\&A 233, 96

Feretti, L., Giovannini, G.: 1996, in Extragalactic Radio Sources, eds. R. Ekers et al., Kluwer, Dordrecht, 333

Feretti, L., Dallacasa, D., Giovannini, G., Tagliani, A.: 1995, A\&A 302,680

Feretti, L., Giovannini, G., Klein, U., et al.: 1998, A\&A 331, 475

Fitt, A. J., Alexander, P.: 1993, MNRAS 261, 445

Fletcher, A., Beck, R., Berkhuijsen, E. M., et al.: 2004, in How Does the Galaxy Work?, eds. E. J. Alfaro et al., Kluwer, Dordrecht, 299

Fletcher, A., Beck, R., Berkhuijsen, E. M., et al.: 2005, A\&A, in prep.

Fürst, E., Reich, W.: 2004, in The Magnetized Interstellar Medium, eds. B. Uyanıker et al., Copernicus, Katlenburg-Lindau, 141

Fujita, Y., Takizawa, M., Sarazin, C. L.: 2003, ApJ 584, 190

Fulks, G. J.: 1975, J. Geophys. Res. 80, 1701

Fusco-Femiano, R., Dal Fiume, D., Feretti, L., et al.: 1999, ApJ 513, L21

Gabici, S., Blasi, P.: 2003, ApJ 583, 695

Giovannini, G., Feretti, L., Venturi, T., Kim, K.-T., Kronberg, P. P.: 1993, ApJ 406, 399

Govoni, F., Feretti, L.: 2004, Int. J. Mod. Phys. D. 13, 1549

Han, J. L., Beck, R., Berkhuijsen, E. M.: 1998, A\&A 335, 1117

Hanasz, M., Lesch, H.: 2003, A\&A 404, 389

Hardcastle, M. J.: 2001, A\&A 373, 881

Hardcastle, M. J., Birkinshaw, M., Worrall, D. M.: 2001, MNRAS 323, L17

Hardcastle, M. J., Birkinshaw, M., Cameron, R. A., et al.: 2002, ApJ 581,948

Harris, D. E., Grindlay, J. E.: 1979, MNRAS 188, 25

Harris, D. E., Carilli, C. L., Perley, R. A.: 1994, Nat 367, 713

Harris, D. E., Nulsen, P. E. J., Ponman, T. J., et al.: 2000, ApJ 530, L81

Heiles, C.: 1976, ARA\&A 14, 1

Helou, G., Bicay, M. D.: 1993, ApJ 415, 93

Hunt, L. K., Dyer, K. K., Thuan, T. X., Ulvestad, J. S.: 2004, ApJ 606,853

Ip, W.-H., Axford, W. I.: 1985, A\&A 149, 7

Kirk, J. G., Guthmann, A. W., Gallant, Y. A., Achterberg, A.: 2000, ApJ 542, 235

Klein, U., Wielebinski, R., Morsi, H. W.: 1988, A\&A 190, 41

Krause, M., Beck, R., Klein, U.: 1984, A\&A 138, 385

Lagage, P. O., Cesarsky, C. J.: 1983, A\&A 125, 249

Lang, K. R.: 1999, Astrophysical Formulae, 3rd edition, Springer, Berlin, Vol. 1
Leahy, J. P., Gizani, N., Tsakiris, D.: 2001, in Particles and Fields in Radio Galaxies, eds. R. A. Laing \& K. M. Blundell, ASP Conf. Ser. 250, 345

Lerche, I., Schlickeiser, R.: 1982, A\&A 107, 148

Lieu, R., Ip, W.-H., Axford, W. I., Bonamente, M.: 1999, ApJ 510, L25

Longair, M. S.: 1994, High Energy Astrophysics, 2nd edition, Cambridge Univ. Press, Vol. 2

Mack, K.-H., Klein, U., O’Dea, C. P., et al.: 1998, A\&A 329, 431

Meisenheimer, K., Röser, H.-J., Hiltner, P. R., et al.: 1989, A\&A 219, 63

Murgia, M., Govoni, F., Feretti, L., et al.: 2004, A\&A 424, 429

Newman, W. I., Newman, A. L., Rephaeli, Y.: 2002, ApJ 575, 755

Niklas, S.: 1995, PhD thesis, University of Bonn

Niklas, S., Beck, R.: 1997, A\&A 320, 54

Niklas, S., Klein, U., Wielebinski, R.: 1997, A\&A 322, 19

Pacholczyk, A. G.: 1970, Radio Astrophysics, Freeman and Co., San Francisco

Parma, P., Murgia, M., Morganti, R., et al.: 1999, A\&A 344, 7

Pérez-Torres, M. A., Alberdi, A., Marcaide, J. M., et al.: 2002, MNRAS 335, L23

Pfrommer, C., Enßlin, T. A.: 2004a, A\&A 413, 17

Pfrommer, C., Enßlin, T. A.: 2004b, MNRAS 352, 76

Pohl, M.: 1993a, A\&A 270, 91

Pohl, M.: 1993b, A\&A 279, L17

Rees, M.: 1985, in Extragalactic Energetic Sources, ed. V. K. Kapahi, Ind. Acad. of Sci., Bangalore, 53

Reich, P., Reich, W.: 1988, A\&A 196, 211

Roettiger, K., Stone, J. M., Burns, J. O.: 1999, ApJ 518, 594

Rohlfs, K., Wilson, T. L.: 1996, Tools of Radio Astronomy, 2nd edition, Springer, Berlin, 248

Sarazin, C. L.: 1999, ApJ 520, 529

Schlickeiser, R.: 2002, Cosmic Ray Astrophysics, Springer, Berlin

Schlickeiser, R., Fürst, E.: 1989, A\&A 219, 192

Schlickeiser, R., Miller, J. A.: 1998, ApJ 492, 352

Segalovitz, A., Shane, W. W., de Bruyn, A. G.: 1976, Nat 264, 222

Strong, A. W., Moskalenko, I. V., Reimer, O.: 2000, ApJ 537, 763

Tashiro, M., Kaneda, H., Makishima, K., et al.: 1998, ApJ 499, 713

Thierbach, M., Klein, U., Wielebinski, R.: 2003, A\&A 397, 53

Verschuur, G. L.: 1979, Fund. Cosmic Phys. 5, 113

Völk, H. J., Zank, L. A., Zank, G. P.: 1988, A\&A 198, 274

Webber, W. R., Simpson, G. A., Cane, H. V.: 1980, ApJ 236, 448

Wilson, A. S., Young, A. J., Shopbell, P. L.: 2000, ApJ 544, L27

Wilson, A. S., Young, A. J., Shopbell, P. L.: 2001, ApJ 547, 740

\section{Appendix A: The revised formula for energy equipartition or minimum energy}

In all electromagnetic acceleration models, like the first-order and second-order Fermi shock acceleration (Drury 1983, Schlickeiser 2002, Gabici \& Blasi 2003) and the acceleration by MHD waves (Schlickeiser \& Miller 1998, Brunetti et al. 2001b, Fujita et al.2003), the energy spectrum of cosmic rays is a power law in momentum which translates into a power law in particle energy $E$ with a break at $E_{\mathrm{b}}=m c^{2}$, where $m$ is the rest mass of the accelerated particle (Bell 1978):

$$
\begin{aligned}
n(E) d E= & n_{0}\left(E_{\mathrm{b}} / E_{0}\right)^{\left(1-\gamma_{0}\right) / 2}\left(E / E_{0}\right)^{-\left(\gamma_{0}+1\right) / 2} d E \\
& \left(E_{1}<E<E_{\mathrm{b}}\right) \\
n(E) d E= & n_{0}\left(E / E_{0}\right)^{-\gamma_{0}} d E \\
& \left(E>E_{\mathrm{b}}\right)
\end{aligned}
$$


$n(E)$ is the number of cosmic ray particles per unit volume and per unit energy interval. $E_{0}\left(E_{0}>E_{\mathrm{b}}\right)$ is an energy normalization to obtain correct units; $n_{0}=n\left(E_{0}\right) ; E_{0}=1$ erg if all calculations are performed in cgs units. $\gamma_{0}$ is the injection spectral index of the energy spectrum. $E_{1}$ is the threshold energy for acceleration (see below).

The solution of the transport equations for Fermi acceleration in a strong, non-magnetic shock in a non-relativistic gas (compression ratio $r=4$ ) yields an injection spectral index of $\gamma_{0}=(r+2) /(r-1)=2.0$ and $\gamma_{0}=2.5$ in a relativistic gas (compression ratio $r=3$ ) (Drury 1983, Schlickeiser 2002). The limit for ultrarelativistic shocks is $\gamma_{0}=2.23$ (Kirk et al. 2000). In a low- $\beta$ plasma (i.e a dominant magnetic field, where $\beta$ is the ratio of thermal to magnetic energy densities) the compression is larger and $\gamma_{0}$ can be flatter than 2 (Schlickeiser \& Fürst 1989). If $\beta$ is close to unity, the injection spectral index is $\gamma_{0} \simeq 2$, almost independent of the compression ratio.

In models of first-order Fermi acceleration in supernova shocks the effective injection spectral index of protons (and probably also electrons) is $\gamma_{0} \simeq 2.1-2.3$ (Bogdan \& Völk 1983, Völk et al. 1988) which agrees well with the source spectrum fitted to the observations of local Galactic cosmic ray nuclei near the sun (Engelmann et al.1990). Intergalactic shocks due to merging clusters produce electron spectra with $\gamma_{0} \simeq 2.3-2.4$ (Gabici \& Blasi 2003).

The total number of cosmic ray particles is (for $\gamma>1$ and negligible energy losses):

$$
\begin{aligned}
N & =\int_{E_{1}}^{\infty} n(E) d E \\
& =\frac{n_{0} E_{0}}{\gamma_{0}-1}\left[2\left(E_{\mathrm{b}} E_{1} / E_{0}^{2}\right)^{\left(1-\gamma_{0}\right) / 2}-\left(E_{\mathrm{b}} / E_{0}\right)^{\left(1-\gamma_{0}\right)}\right] \\
& \approx \frac{2 n_{0} E_{0}}{\gamma_{0}-1}\left(E_{0}^{2} / E_{\mathrm{b}} E_{1}\right)^{\left(\gamma_{0}-1\right) / 2}
\end{aligned}
$$

where the approximation is valid for $E_{1} \ll E_{\mathrm{b}}$.

We adopt the commonly used assumption that the same total numbers of non-relativistic protons and electrons are accelerated above a certain energy threshold $E_{1}$, e.g. the nonrelativistic kinetic energy of $E_{1} \simeq 10 \mathrm{KeV}$. From this requirement it follows that the ratio of number densities at energy $E_{0}$ $\left(E_{0}>E_{\mathrm{p}}\right)$ has to be:

$n_{\mathrm{p}, 0} / n_{\mathrm{e}, 0}=\left(E_{\mathrm{p}} / E_{\mathrm{e}}\right)^{\left(\gamma_{0}-1\right) / 2}$

where $E_{\mathrm{p}}$ and $E_{\mathrm{e}}$ are the spectral break energies for protons and electrons, respectively.

$\left(E_{\mathrm{p}}=938.28 \mathrm{MeV}=1.5033 \cdot 10^{-3} \mathrm{erg}\right.$ and $E_{\mathrm{e}}=511 \mathrm{keV}$ $=8.187 \cdot 10^{-7} \mathrm{erg}$ )

The total energy density of cosmic ray particles (for negligible energy losses) is:

$$
\begin{aligned}
\epsilon= & \int_{E_{1}}^{\infty} n(E) E d E \\
= & \int_{E_{1}}^{E_{\mathrm{b}}} n_{0}\left(E_{\mathrm{b}} / E_{0}\right)^{\left(1-\gamma_{0}\right) / 2} E_{0}\left(E / E_{0}\right)^{\left(1-\gamma_{0}\right) / 2} d E \\
& +\int_{E_{\mathrm{b}}}^{\infty} n_{0} E_{0}\left(E / E_{0}\right)^{1-\gamma_{0}} d E
\end{aligned}
$$

For $E_{1} \ll E_{\mathrm{b}}$ and $2<\gamma_{0}<3$, the high-energy particles carry the total energy and Eq. A5 gives:

$\epsilon=n_{0} E_{0}^{2}\left(E_{0} / E_{\mathrm{b}}\right)^{\gamma_{0}-2}\left(\frac{2}{3-\gamma_{0}}+\frac{1}{\gamma_{0}-2}\right)$

For $\gamma_{0}=2$ (very strong shocks):

$\epsilon=n_{0} E_{0}^{2}\left(2+\ln \left(E_{2} / E_{\mathrm{b}}\right)\right)$

where $E_{2}$ is the high-energy limit of the spectrum according to diffusion coefficient and/or the finite age of the acceleration region (Lagage \& Cesarsky 1983). The $\mathrm{TeV} \gamma$-ray image of a supernova remnant confirmed the existence of particles of very high energies (Aharonian et al. 2004). For $\gamma_{0}=3$ :

$\epsilon=n_{0} E_{0}^{2}\left(E_{0} / E_{\mathrm{b}}\right)\left(1+\ln \left(E_{\mathrm{b}} / E_{1}\right)\right)$

and for $\gamma_{0}>3$ (weak shocks) the low-energy particles carry the total energy:

$$
\begin{aligned}
\epsilon= & n_{0} E_{0}^{2}\left(E_{0} / E_{\mathrm{b}}\right)^{\left(\gamma_{0}-1\right) / 2}\left(E_{0} / E_{1}\right)^{\left(\gamma_{0}-3\right) / 2} \\
& \times\left(\frac{2}{3-\gamma_{0}}\right)
\end{aligned}
$$

As a consequence of the different rest masses of protons and electrons, their spectral break energy $E_{\mathrm{b}}$ differs, and hence the energy spectrum of electrons steepens at lower energies than that of protons (see Fig. 1 in Pohl 1993a). The proton number per energy interval dominates for $E>E_{\mathrm{e}}=$ $511 \mathrm{keV}$ (see Eqs. (7) and (10).

Eq. (A6) is valid only for the injection spectrum. The observed spectral index beyond $10 \mathrm{GeV}$ is $\gamma \simeq 2.7$ for many cosmic ray species near the sun (Fulks 1975) and in spiral galaxies (Niklas et al. 1997), which is steeper than the injection spectrum and indicates an energy-dependent escape time from the Milky Way of $t_{\text {esc }} \propto E^{-0.6}$ (Engelmann et al. 1990). Furthermore, the sub-relativistic cosmic ray protons suffer from ionization and/or Coulomb losses, so that the spectrum becomes almost flat at energies below $E_{\mathrm{p}}$ with an energy spectral index of $\left(2-\gamma_{\mathrm{p}, 0}\right) / 2 \simeq 0.1-0.2$ (e.g. Pohl 1993a). The observed spectrum of local Galactic protons, corrected for modulation by the solar wind, gradually steepens between $100 \mathrm{MeV}$ and $10 \mathrm{GeV}$ (Fulks 1975, Ip \& Axford 1985) and can be described as:

$$
\begin{aligned}
n(E) d E \cong & \text { const } d E=n_{\mathrm{p}, 0}\left(E_{\mathrm{p}} / E_{0}\right)^{-\gamma_{\mathrm{p}}} d E \\
& (E \lesssim 100 \mathrm{MeV}) \\
n(E) d E= & n_{\mathrm{p}, 0}\left(E / E_{0}\right)^{-\gamma_{\mathrm{p}}} d E \\
& (E \gtrsim 10 \mathrm{GeV})
\end{aligned}
$$

with $\gamma_{\mathrm{p}} \simeq 2.7$. A similar spectral behaviour of the protons is expected in all objects containing ionized or neutral gas, so that Eq. A11 is of general applicability. Replacing the observed gradual steepening around $E_{\mathrm{p}}$ by a break at $E_{\mathrm{p}}$, the energy density of the protons is approximated by:

$$
\begin{aligned}
\epsilon_{\mathrm{p}} \cong & \int_{E_{1}}^{E_{\mathrm{p}}} n_{\mathrm{p}, 0}\left(E_{\mathrm{p}} / E_{0}\right)^{-\gamma_{\mathrm{p}}} E d E \\
& +\int_{E_{\mathrm{p}}}^{\infty} n_{\mathrm{p}, 0} E_{0}\left(E / E_{0}\right)^{1-\gamma_{\mathrm{p}}} d E
\end{aligned}
$$


For $E_{1} \ll E_{\mathrm{p}}$ and $\gamma_{\mathrm{p}}>2$ :

$\epsilon_{\mathrm{p}}=n_{\mathrm{p}, 0} E_{0}^{2}\left(E_{0} / E_{\mathrm{p}}\right)^{\gamma_{\mathrm{p}}-2}\left(\frac{1}{2}+\frac{1}{\gamma_{\mathrm{p}}-2}\right)$

For very strong shocks which generate injection spectra with $\gamma_{p}=2.0$ (Sect. 3.2, Eq. A13) has to be replaced by:

$\epsilon_{\mathrm{p}}=n_{\mathrm{p}} E_{0}^{2}\left(\frac{1}{2}+\ln \left(E_{2} / E_{\mathrm{p}}\right)\right)$

The scaling parameter $n_{\mathrm{p}, 0}$ of the proton spectrum can be determined in "thin targets" from that of the electron spectrum $n_{\mathrm{e}, 0}$ and the proton-to-electron number density ratio $\mathbf{K}_{\mathbf{0}}$, and $n_{\mathrm{e}, 0}$ can be replaced by the synchrotron intensity (surface brightness) $I_{\nu}$ (in erg s $\mathrm{cm}^{-1} \mathrm{~Hz}^{-1} \mathrm{sterad}^{-1}$ ), observed at the frequency $\nu$, and the magnetic field strength $B_{\perp}$ in the sky plane (in G), making use of the synchrotron formula (e.g. Pacholczyk 1970):

$I_{\nu}=c_{2}\left(\gamma_{\mathrm{e}}\right) n_{\mathrm{e}, 0} E_{0}^{\gamma_{\mathrm{e}}} l\left(\nu / 2 c_{1}\right)^{\left(1-\gamma_{\mathrm{e}}\right) / 2} B_{\perp}^{\left(\gamma_{\mathrm{e}}+1\right) / 2}$

where

$$
\begin{aligned}
c_{1}= & 3 e /\left(4 \pi m_{\mathrm{e}}{ }^{3} c^{5}\right)=6.26428 \cdot 10^{18} \mathrm{erg}^{-2} \mathrm{~s}^{-1} \mathrm{G}^{-1}, \\
c_{2}\left(\gamma_{\mathrm{e}}\right)= & \frac{1}{4} c_{3}\left(\gamma_{\mathrm{e}}+7 / 3\right) /\left(\gamma_{\mathrm{e}}+1\right) \Gamma\left[\left(3 \gamma_{\mathrm{e}}-1\right) / 12\right] \\
& \times \Gamma\left[\left(3 \gamma_{\mathrm{e}}+7\right) / 12\right] \\
c_{3}= & \sqrt{3} e^{3} /\left(4 \pi m_{\mathrm{e}} c^{2}\right) \\
= & 1.86558 \cdot 10^{-23} \mathrm{erg} \mathrm{G}^{-1} \mathrm{sterad}^{-1}
\end{aligned}
$$

$c_{2}\left(\gamma_{\mathrm{e}}\right)$ is identical to $c_{5}\left(\gamma_{\mathrm{e}}\right)$ in Pacholczyk (1970 p. 95 and tabulated on p. 232). $n_{\mathrm{e}}(E)$ is the number density of cosmic ray electrons per unit energy interval (in $\mathrm{cm}^{-3} \mathrm{erg}^{-1}$ ) in the relevant energy range. (Note that Pacholczyk (1970) used a different definition of $n_{\mathrm{e}}(E)$ which leads to strange units.) $l$ is the pathlength through the emitting medium. The spectral index $\alpha$ of the synchrotron emission relates to the spectral index $\gamma_{\mathrm{e}}$ of the energy spectrum of the electrons via $\alpha=$ $\left(\gamma_{\mathrm{e}}-1\right) / 2$.

We define $E_{\text {syn }}$ as the electron energy beyond which synchrotron and inverse Compton losses are significant, hence the ratio $\mathbf{K}(\mathrm{E})$ is not constant, and the equipartition estimate fails (see Sect. 3.4). In the energy range $E_{\mathrm{p}}<E<E_{\text {syn }}$, the ratio is constant $\left(\mathbf{K}=\mathbf{K}_{\mathbf{0}}\right)$ and the proton and electron spectra are power laws with the same spectral index $\left(\gamma_{\mathrm{e}}=\gamma_{\mathrm{p}}=\gamma\right)$ if nonthermal bremsstrahlung loss of the electrons is negligible (see Sect. 3.4). Then the radio synchrotron spectrum can be used to extrapolate the proton spectrum, so that $n_{\mathrm{p}}$ in Eq. $\mathrm{A13}$ can be replaced by:

$n_{\mathrm{p}, 0}=\mathbf{K}_{\mathbf{0}} I_{\nu}\left(\nu / 2 c_{1}\right)^{(\gamma-1) / 2} /\left[c_{2}(\gamma) E_{0}^{\gamma} l B_{\perp}^{(\gamma+1) / 2}\right]$

and the total cosmic ray energy density becomes:

$$
\begin{aligned}
\epsilon_{\mathrm{CR}}= & \gamma\left(\mathbf{K}_{\mathbf{0}}+1\right) I_{\nu} E_{\mathrm{p}}^{2-\gamma}\left(\nu / 2 c_{1}\right)^{(\gamma-1) / 2} \\
& /\left[2(\gamma-2) c_{2}(\gamma) l B_{\perp}^{(\gamma+1) / 2}\right]
\end{aligned}
$$

Note that, according to Eq. $\mathrm{A6}, \epsilon_{\mathrm{CR}}$ includes protons up to the highest energies.
The projected field component $B_{\perp}$ has to be replaced by the total field $B$ :

$B_{\perp}^{(\gamma+1) / 2}=B^{(\gamma+1) / 2} c_{4}(i)$

$c_{4}(i)=[\cos (i)]^{(\gamma+1) / 2}$ is valid for observation of a region where the field is completely regular and has a constant inclination $i$ with respect to the sky plane $\left(i=0^{\circ}\right.$ is the face-on view), e.g. in a small region of the Milky Way or a galaxy. If the field is completely turbulent and has an isotropic angle distribution in three dimensions, $B_{\perp}^{2}=(2 / 3) B^{2}$ and $c_{4}(i)=(2 / 3)^{(\gamma+1) / 4}$. If the synchrotron intensity is averaged over a large volume, $[\cos (i)]^{(\gamma+1) / 2}$ has to be replaced by its average over all occuring values of $i$. The case of a field oriented parallel to a galactic disk was treated by Segalovitz et al. (1976 their Eq. (3)).

Combining $\epsilon_{\mathrm{CR}}=\epsilon_{\mathrm{B}}=B_{\mathrm{eq}}^{2} / 8 \pi$ with Eq. A16 and replacing $\gamma$ by $(2 \alpha+1)$ leads to:

$$
\begin{aligned}
B_{\mathrm{eq}}= & \left\{4 \pi(2 \alpha+1)\left(\mathbf{K}_{\mathbf{0}}+1\right) I_{\nu} E_{\mathrm{p}}^{1-2 \alpha}\left(\nu / 2 c_{1}\right)^{\alpha}\right. \\
& \left./\left[(2 \alpha-1) c_{2}(\alpha) l c_{4}(i)\right]\right\}^{1 /(\alpha+3)}
\end{aligned}
$$

For use in the classical formula, Eq. A17 has to be replaced by:

$B_{\perp}^{3 / 2}=B^{3 / 2} c_{4}(i)$

With the definitions introduced before, we can write the classical minimum-energy formula (1) as:

$$
\begin{aligned}
B_{\text {class }}= & \left\{6 \pi ( \mathcal { K } + 1 ) I _ { \nu } ( \nu / 2 ) ^ { \alpha } c _ { 1 } ^ { - 1 / 2 } \left[\nu_{\min }^{(1 / 2-\alpha)}\right.\right. \\
& \left.\left.-\nu_{\max }^{(1 / 2-\alpha)}\right] /\left[(2 \alpha-1) c_{2}(\alpha) l c_{4}(i)\right]\right\}^{2 / 7}
\end{aligned}
$$

A table to compute $B_{\text {class }}$ from $I_{\nu}$ (in mJy/arcsec ${ }^{2}$ ) and $l$ (in $\mathrm{kpc}$ ) is given in Govoni \& Feretti (2004).

As discussed in Sect. 2 the integration of the radio spectrum between the fixed frequencies $\nu_{\min }$ and $\nu_{\max }$ introduces an implicit dependence on field strength $B$ which is ignored when computing the derivative $d \epsilon_{\text {tot }} / d B$. The constant exponent 2/7 in Eq. A20 is the result of this error, which is one of the reasons why the classical formula gives wrong results (see Sect. 2). 